\title{
Optical phase conjugation for nonlinearity compensation in WDM PDM 16-QAM transmission over dispersion-compensated and dispersion-uncompensated links
}

Da Ros, Francesco; Sackey, I.; Jazayerifar, M.; Richter, T.; Elschner, Robert; Meuer, C.; Noelle, Markus; Molle, Lutz; Petermann, Klaus; Fischer, J.K.

Total number of authors:

12

Published in:

Proceedings of 2015 IEEE Photonics Society Summer Topicals Meeting Series

Link to article, DOI:

10.1109/PHOSST.2015.7248199

Publication date:

2015

Document Version

Peer reviewed version

Link back to DTU Orbit

Citation (APA):

Da Ros, F., Sackey, I., Jazayerifar, M., Richter, T., Elschner, R., Meuer, C., Noelle, M., Molle, L., Petermann, K., Fischer, J. K., Schubert, C., \& Peucheret, C. (2015). Optical phase conjugation for nonlinearity compensation in WDM PDM 16-QAM transmission over dispersion-compensated and dispersion-uncompensated links. In Proceedings of 2015 IEEE Photonics Society Summer Topicals Meeting Series (pp. 72-73). IEEE. https://doi.org/10.1109/PHOSST.2015.7248199

\section{General rights}

Copyright and moral rights for the publications made accessible in the public portal are retained by the authors and/or other copyright owners and it is a condition of accessing publications that users recognise and abide by the legal requirements associated with these rights.

- Users may download and print one copy of any publication from the public portal for the purpose of private study or research.

- You may not further distribute the material or use it for any profit-making activity or commercial gain

- You may freely distribute the URL identifying the publication in the public portal 


\title{
Optical phase conjugation for nonlinearity compensation in WDM PDM 16-QAM transmission over dispersion-compensated and dispersion-uncompensated links
}

\author{
F. Da $\operatorname{Ros}^{1}$, I. Sackey ${ }^{2,3}$, M. Jazayerifar ${ }^{2}$, T. Richter ${ }^{3}$, R. Elschner ${ }^{3}$, C. Meuer ${ }^{2,3}$, \\ M. Nölle ${ }^{3}$, L. Molle ${ }^{3}$, K. Petermann ${ }^{2}$, J.K. Fischer ${ }^{3}$ C. Schubert ${ }^{3}$, and C. Peucheret ${ }^{4}$ \\ ${ }^{1}$ Department of Photonics Engineering, Technical University of Denmark, DK-2800 Kgs. Lyngby, Denmark \\ ${ }^{2}$ Technische Universität Berlin, Fachgebiet Hochfrequenztechnik, 10587 Berlin, Germany \\ ${ }^{3}$ Fraunhofer Institute for Telecommunications, Heinrich Hertz Institute, 10587 Berlin, Germany \\ ${ }^{4}$ FOTON Laboratory, CNRS UMR 6082, ENSSAT, University of Rennes 1, F-22305 Lannion, France \\ e-mail: fdro@fotonik.dtu.dk
}

\begin{abstract}
Kerr nonlinearity compensation by optical phase conjugation is demonstrated in a WDM PDM 16-QAM system. Improved received signal quality is reported for both dispersion-compensated and dispersion-uncompensated transmission and a comparison with digital backpropagation is provided.
\end{abstract}

Keywords-nonlinear optical signal processing; four-wave mixing; optical phase conjugation, nonlinearity compensation.

\section{INTRODUCTION}

Increasingly complex modulation formats are being used in optical communication systems in order to keep up with the demand for capacity. This has the undesired effect of increasing the required optical signal-to-noise ratio (OSNR) for error-free transmission, as well as decreasing the tolerance to transmission impairments [1]. Injecting higher optical power per wavelength channel in the transmission link improves the OSNR. Kerr nonlinearity, however, sets an upper bound to the power that can be transmitted before degrading the signal performance by nonlinear distortion [2]. Various techniques aim at mitigating transmission impairments caused by the Kerr nonlinearity and are thus being investigated both in the digital [3-7] and optical [8-16] domain.

In principle all-optical nonlinearity mitigation has the appealing advantage of providing real-time compensation for entire wavelength-division multiplexed (WDM) signals without requiring additional complex electronic circuitry necessary for digital signal processing techniques such as digital backpropagation (DBP).

Optical phase conjugation (OPC), in particular, is a well-known method used to provide dispersion compensation by lumped mid-span spectral inversion (MSSI). Provided the transmission link possesses a sufficient degree of symmetry in dispersion and power distribution with respect to the OPC position, Kerr nonlinearity compensation can be achieved [8].

In this contribution we review some of our recent experimental results on using OPC to compensate for nonlinear distortions in a 5-channel WDM polarization-division multiplexed (PDM) 16-ary quadrature amplitude modulation (16-QAM) transmission system. A fiber-based OPC device has been optimized and two different link configurations have been investigated: dispersion-compensated transmission with lumped amplification using erbium-doped fiber amplifiers (EDFAs) $[10,11]$ and dispersion-uncompensated transmission utilizing distributed Raman amplification (DRA) [12,13]. A significant increase in the received Q-factor and transmission reach compared to straight transmission is demonstrated for both configurations. Additionally, a comparison between OPC and single-channel DBP based on the split-step Fourier method (SSFM) is reported.

\section{OPTICAL PHASE CONJUGATOR}

In order to be suitable for operation in an optical network, an OPC device should fulfill some basic requirements such as transparency to bitrate and modulation format, polarizationinsensitive operation and ideally causing no performance deterioration to the WDM signal.

The black-box OPC device used in our demonstrations is based on a dual-pump polarization-insensitive scheme relying on a polarization diversity loop with a highly nonlinear fiber (HNLF) as nonlinear medium, as shown in Fig. $1[11,12]$. The setup exhibits less than $0.3 \mathrm{~dB}$ of polarization-dependent gain [12].

In order to provide positive conversion efficiency while minimizing the degradation of the conjugated idler due to pump phase noise transfer, the two continuous wave (CW) pumps (1534 nm and $1574 \mathrm{~nm}$ ) generated from external cavity lasers (ECLs) are phase modulated (PM) in counter-phasing operation by two frequency tones at $69 \mathrm{MHz}$ and $253 \mathrm{MHz}$ with a modulation index of 1.44 radians.

The signal input power and on-off gain have been optimized to minimize the OSNR penalty introduced by the OPC operation to below $1 \mathrm{~dB}$. A total WDM signal power of $2 \mathrm{dBm}$ and a total pump power of $29.2 \mathrm{dBm}$ (5-dB on-off gain) at the polarization-diversity loop input was used $[11,12]$.

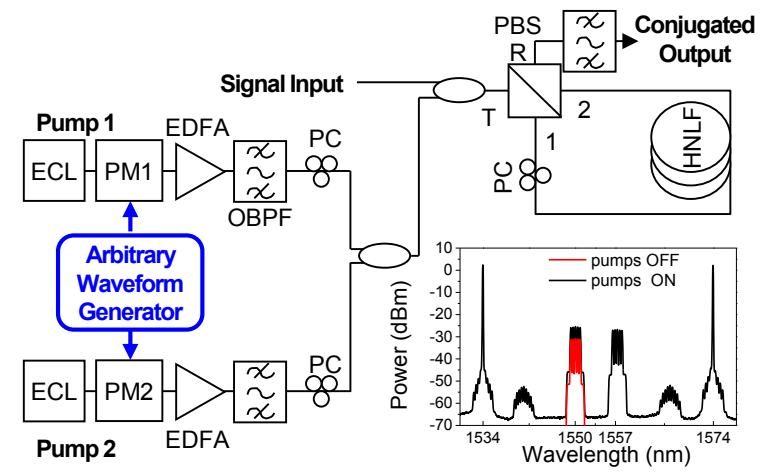

Figure 1. OPC device based on a dual-pump polarization-insensitive scheme: the inset shows the spectra at the polarization-diversity loop output (port R of the polarization beam splitter(PBS)) with pumps OFF and ON. 


\section{KERR NONLINEARITY COMPENSATION}

Using such an optimized OPC device, we experimentally demonstrated Kerr nonlinearity compensation for a 5-channel 28-Gbaud WDM PDM 16-QAM signal transmitted over both dispersion-compensated [10,11] and dispersion-uncompensated links [13].

The dispersion-compensated transmission spans were based on super-large area fiber (SLA) and inverse-dispersion fiber (IDF) with lumped EDFA amplification. The dispersion-uncompensated link consisted of standard singlemode fiber (SSMF) and DRA with backward pumping. While in the first case the link symmetry required for nonlinear compensation was inherently satisfied by the periodic dispersion compensation, the use of DRA is required in the latter scenario to approximate a symmetric signal power profile per span [14].

The received Q-factor, derived from the measured bit-error ratio (BER), for the center (worst) channel is shown as a function of the power launched into each span for the two scenarios in Fig. 2. In both cases application of the OPC device increases the optimum transmission power as well as the maximum received Q-factor with improvements of around $0.9 \mathrm{~dB} \quad(800-\mathrm{km}$ transmission) and $1.1 \mathrm{~dB} \quad(400-\mathrm{km}$ transmission) for the two link configurations. More interestingly, for the dispersion-compensated link, the use of OPC for $800-\mathrm{km}$ transmission results in the same performance as $640-\mathrm{km}$ straight transmission, increasing the reach by $25 \%$.
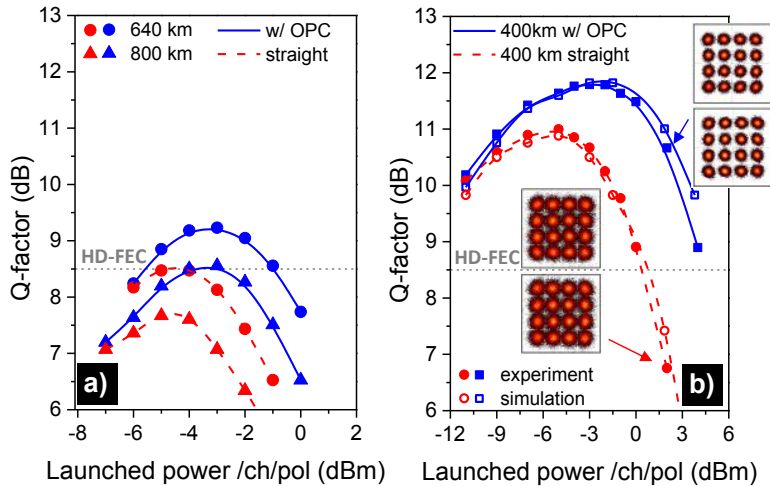

Figure 2. Center channel received Q-factor as a function of the launched power for WDM transmission: (a) experimental results for dispersioncompensated (+EDFA) and (b) experimental and numerical results for dispersion-uncompensated (+DRA) link. Performance with and without (i.e. straight) $\mathrm{OPC}$ is reported. Inset in (b): constellation diagrams at $+2 \mathrm{dBm}$ of launched power; both polarizations are shown.

For the dispersion-uncompensated scenario, numerical simulations, using a link model accurately fitted to the experimental results (as shown in Fig. 2(b)), estimated a reach extension above $80 \%$, from $1300 \mathrm{~km}$ to $2400 \mathrm{~km}$, as shown in Fig. 3(a) [13].

Finally, for $800-\mathrm{km}$ dispersion-compensated transmission, the all-optical approach is compared with single-channel DBP based on SSFM [4]. While DBP provides a higher performance improvement than OPC in a single-channel (SC) scenario (Fig. 3(b)), the advantage of OPC becomes apparent for WDM applications (Fig. 3(c)) [11].

Even though the main results discussed here have been achieved using an HNLF as nonlinear medium, alternative media may allow for smaller footprints and a higher degree of integration. Periodically-poled lithium niobate (PPLN) has been the medium of choice for several demonstrations $[15,16]$. Recently, effective Kerr nonlinearity compensation for WDM QPSK signals has been reported using a silicon nanowire as nonlinear medium [17]. While this demonstration was limited by the still low conversion efficiency in silicon, techniques capable to tackle this issue are being investigated [18], with the potential to provide smaller and more power efficient OPC devices.
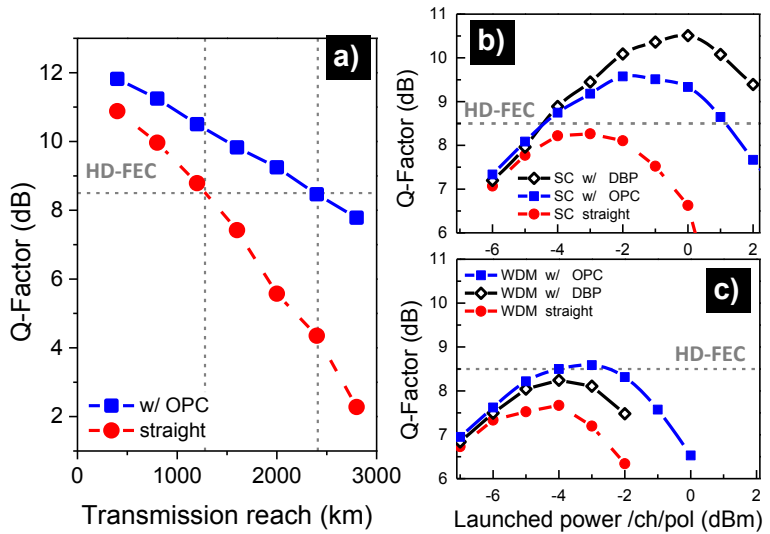

Figure 3. (a) Numerically estimated Q-factor as a function of transmission reach w/ and w/o (i.e. straight) OPC for the dispersion-uncompensated link. Experimental results: received Q-factor as a function of the launched power for (b) single-channel and (c) WDM 800-km dispersion-compensated

transmission: comparison between straight, OPC and DBP cases.

\section{CONCLUSION}

Kerr nonlinearity compensation using optical phase conjugation is demonstrated for a 5-channel WDM PDM 16-QAM transmission over both dispersion-compensated and dispersion-uncompensated links. An extension of the transmission reach is shown for both scenarios and a comparison with digital backpropagation is reported.

\section{ACKNOWLEDGMENT}

This work was funded by the German Research Foundation (DFG) under projects GR 3774/1-1 and PE 319/26-1 and from the Danish Research Council for Technology and Production Sciences under project 09-066562.

\section{REFERENCES}

[1] A.D. Ellis, et al., J. Lightw. Technol., 28, 423-433 (2010).

[2] A.R. Chraplyvy, J. Lightw. Technol, 8,1548-1557 (1990).

[3] R.J. Essiambre and P. Winzer, ECOC, paper Tu.3.2.2 (2005).

[4] E. Ip, JLT 28, 939-951 (2010).

[5] D.S. Millar, et al., JSTQE 16, 1217-1226 (2010).

[6] D. Rafique, A. Ellis, Opt. Express, 19, 16919-16926 (2011).

[7] A. Napoli, et al., J. Lightw. Technol., 32, 1351-1362 (2014).

[8] S. Watanabe, M. Shirasaki, JLT 14, 243-248 (1996).

[9] M.F.C. Stephens, et al., Opt. Express 22, 11840-11848 (2014).

[10] F. Da Ros, et al., ECOC, paper P.5.3, (2014).

[11] I. Sackey, et al., J. Lightw. Technol., pre-print (2015).

[12] I. Sackey, et al., ECOC, paper Tu.1.4.4, (2014).

[13] I. Sackey, et al., Opt. Express, 22, 27381-27391 (2014).

[14] I. Phillips, et al., OFC, paper M3C.1 (2014).

[15] A. Chowdhury, et al., J. Lightw. Technol., 23, 172-177 (2005)

[16] P. Minzioni, Fiber Integrated Opt., 28, 179-209 (2009).

[17] D. Vukovic, et al., Opt. Express, 23, 3640-3646 (2015).

[18] A. Gajda, et al., Opt. Express, 20, 13100-13107 (2010) 